\title{
COSTOS DE CUMPLIMIENTO DE REGULACIÓN AMBIENTAL CON INFORMACIÓN INCOMPLETA. APLICACIÓN A FUENTES FIJAS DEL PCE DE SANTIAGO, CHILE*
}

\author{
COMPLIANCE COSTS OF ENVIRONMENTAL \\ REGULATION WITH INCOMPLETE INFORMATION. \\ APPLICATION TO FIXED SOURCES OF PCE IN SANTIAGO, CHILE
}

\author{
Gaspard Clerger**, Carlos Chávez***, \\ Mauricio Villena****, Walter GómeZ*****
}

\begin{abstract}
Resumen
Estudiamos la propiedad de costo-efectividad de un sistema de permisos de emisión transferibles (SPET) frente un sistema de estándares de emisión. Nuestro análisis agrega a los costos de abatimiento, los costos de fiscalización para inducir cumplimiento. Consideramos, además, escenarios de información completa e incompleta. Las simulaciones numéricas se basan en datos de las fuentes fijas que operan en el Programa de Compensación de Emisiones (PCE) en Santiago de Chile. Los resultados muestran que un SPET no permite obtener mejoras en calidad del aire al mínimo costo de fiscalización, pero mantiene su costo-efectividad en términos de los costos totales de cumplimiento.
\end{abstract}

Palabras clave: Política ambiental, costo-efectividad, costos de fiscalización, información incompleta.

\footnotetext{
* Enviar correspondencia a Carlos Chávez, Profesor Titular, Departamento de Economía, Universidad de Concepción, Casilla 1987, Concepción, Chile, Tel. 56-41-2203067, E-mail: cchavez@udec.cl. Clerger, Chávez y Villena agradecen el apoyo financiero proporcionado por Conicyt-Chile, a través del Proyecto Fondecyt No 1060679 . Gómez agradece el apoyo financiero proporcionado por Conicyt-Chile, a través del Proyecto Fondecyt $\mathrm{N}^{\circ} 1050696$. Chávez y Gómez agradecen también el apoyo financiero proporcionado por Conicyt-Chile, a través del Proyecto Fondecyt $\mathrm{N}^{\circ} 1080287$, que permitió realizar el proceso de revisión de este artículo.

** Programa Magíster en Economía de Recursos Naturales y del Medio Ambiente, Universidad de Concepción.

*** Departamento de Economía, Universidad de Concepción.

**** Escuela de Negocios, Universidad Adolfo Ibáñez.

***** Departamento de Ingeniería Matemática, Universidad de La Frontera.
} 


\begin{abstract}
We study the cost-effectiveness of a transferable emissions permit system (TEPS) vis a vis a system of emissions standards. Our analysis includes along with abatement costs, the costs of enforcing the system to induce compliance. Further, the analysis considers complete and incomplete information. The numerical simulations are performed for the case of fixed sources operating under the Emissions Compensation Program (ECP) in Santiago, Chile. The results suggest that a TEPS is not able to induce compliance at minimum enforcement costs, but this regulatory system allow the regulator to achieve the environmental target with minimum aggregate compliance costs.
\end{abstract}

Key words: Environmental policy, cost-effectiveness, enforcement costs, incomplete information.

JEL Classification: $L 51, Q 28, K 42, K 32$.

\title{
1. InTRODUCCIóN
}

El sistema de permisos de emisión transferibles (SPET) es uno de los instrumentos económicos más innovadores para el control de contaminación atmosférica actualmente disponibles. Estudios basados en la evaluación de los costos agregados de abatimiento asociados a la utilización de un SPET han sugerido su uso para solucionar problemas de contaminación atmosférica de manera costo-efectiva (Arimura, 2002; Burtraw, 1996, 1999; McLean, 1997; Schmalensee et al., 1998; Stavins, 1998) ${ }^{1}$. Esta propiedad de costo-efectividad del SPET no se puede garantizar, en el caso de información completa, si se consideran adicionalmente los costos de fiscalización para inducir cumplimiento (ver por ejemplo Malik, 1992; Hahn y Axtell, 1995, y más recientemente Chávez et al., 2009). Si la información disponible al regulador es incompleta, puede de nuevo asegurarse que el sistema SPET es costo-efectivo respecto a la estrategia de comando y control al considerar costos agregados de abatimiento y fiscalización (Chávez et al., 2009).

Este artículo estudia, mediante simulaciones numéricas, la propiedad de costoefectividad de un SPET frente un sistema de estándares de emisión (EE) cuando se consideran, además de los costos de abatimiento, los costos de fiscalización en contextos de información completa e información incompleta. Los datos para implementar las simulaciones numéricas se refieren a las fuentes fijas reguladas por el Programa de Compensación de Emisiones (PCE) de Santiago de Chile.

Dos elementos motivan el estudio numérico. Primero, desde un punto de vista conceptual, en presencia de información completa no es posible dilucidar si un SPET es más costo-efectivo que una política de comando y control (Chávez et al., 2009; Malik, 1992). Segundo, aun en el caso de información

1 A nivel nacional, véase también O'Ryan (1996), Villena y Villena (1998), O'Ryan y Bravo (2001). 
incompleta, en que se sabe que un SPET es más costo-efectivo que una política de comando y control (Chávez et al., 2009), la simulación permite cuantificar para el caso de estudio, los órdenes de magnitud de la ganancia asociada al uso de SPET sobre una política de comando y control, incluyendo los costos de fiscalización.

Los objetivos específicos de este trabajo son: (i) calcular y comparar mediante simulaciones numéricas, los costos de abatimiento, costos de fiscalización y costos de cumplimiento para cada instrumento de regulación en el marco de fuentes participando en el PCE; y (ii) estudiar a través de un análisis de sensibilidad, el impacto sobre los costos agregados de abatimiento, de fiscalización, y los costos de cumplimiento, de cambios en la meta ambiental, en los parámetros que definen las funciones individuales de costos de abatimiento, sanción por incumplimiento y número de fuentes reguladas.

Este trabajo introduce dos innovaciones con respeto a la literatura empírica relacionada al control de la contaminación atmosférica basada en incentivos económicos. Por una parte, incorporamos en las simulaciones los costos de fiscalización necesarios para implementar un determinado instrumento de control de contaminación. Por otra parte, además de presentar evidencia numérica sobre un resultado teórico reciente (Chávez et al., 2009) en cuanto a la propiedad de costo-efectividad de un SPET sobre una política de comando y control bajo información incompleta, se presenta una cuantificación de esta relación de costo-efectividad para el caso de estudio. Adicionalmente, proveemos un ejemplo en el cual los costos de fiscalización necesarios para inducir cumplimiento en un SPET exceden a aquellos asociados a un instrumento de comando y control con información completa sin afectar la propiedad de costo-efectividad del SPET, lo cual fue sugerido teóricamente por Malik (1992). Como resultado de nuestras simulaciones, también se obtiene una cuantificación del esfuerzo de fiscalización por parte del regulador para inducir perfecto cumplimiento bajo distintos instrumentos y escenarios de regulación.

El artículo está estructurado de la siguiente manera: en la sección 2 se presenta brevemente el modelo teórico considerado en el marco de este artículo. En la sección 3 se describe la metodología utilizada en las simulaciones. En la sección 4 se presentan los principales resultados obtenidos: costos de abatimiento, costos de fiscalización, y costos de cumplimiento para cada instrumento bajo estudio. En la sección 5 se presentan las conclusiones a partir de los resultados obtenidos en las simulaciones.

\section{CUMPLimiento: SPET versus Estándares de Emisión}

En esta sección se presenta brevemente el modelo teórico desarrollado por Chávez et al. (2009) para examinar el costo de cumplimiento de un SPET versus un sistema de estándares de emisión (EE).

\subsection{Cumplimiento individual de las fuentes reguladas}

Consideramos un número total fijo $N$ de firmas heterogéneas y neutrales al riesgo. Indexamos las firmas por $i$ y denotamos por $e_{i}$ el nivel individual de emisiones generadas por la firma $i$, que constituye en este modelo una variable 
de decisión disponible a la firma. La función de costo de abatimiento de la firma $i$ la llamaremos $c_{i}\left(e_{i}\right)$, y la suponemos estrictamente decreciente y convexa en $e_{i}$, o sea $\left[c_{i}^{\prime}\left(e_{i}\right)<0\right]$ y $\left[c_{i}^{\prime \prime}\left(e_{i}\right)>0\right]^{2}$. Esta hipótesis es útil para el desarrollo, pero en algunos casos reales puede ser ciertamente demasiado estricta; ver comentarios al respecto en la sección 3 .

En este trabajo la meta ambiental es determinada por un agente regulador en forma de un nivel agregado de emisiones $E$. El mismo agente fija la regulación bajo la cual operan las firmas, y que puede ser, en nuestro estudio, un estándar de emisión o también un sistema competitivo de permisos de emisión.

En el caso de un estándar de emisión el regulador, teniendo en cuenta la meta ambiental, fija un estándar $s_{i}$ que aplica a cada fuente $i$. Bajo este sistema una violación de emisiones $\left(v_{\mathrm{i}}\right)$ ocurre si las emisiones de la firma sobrepasan el estándar $s_{i}$, o sea $v_{i}=e_{i}-s_{i}>0$. La firma está expuesta a una probabilidad individual de monitoreo $\pi_{i}$. Si la firma es auditada y sorprendida en violación, una multa $f\left(v_{i}\right)$ será impuesta ${ }^{3}$. El problema de la firma es elegir el nivel de emisiones que minimiza sus costos de cumplimiento esperados, los cuales consisten en el costo de abatimiento más una multa esperada. En este modelo, una firma elegirá un nivel de emisiones en cumplimiento con el estándar $s_{i}$, en el caso que se cumpla la siguiente condición (ver Malik, 1992; Heyes, 2000):

$$
-c_{i}^{\prime}\left(s_{i}\right) \leq \pi_{i} f^{\prime}(0)
$$

Entonces, la elección de cumplir de una firma individual requiere que la multa marginal esperada no sea menor que el costo marginal de abatimiento asociado con el nivel de emisiones equivalente al estándar de emisiones ${ }^{4}$.

Bajo un SPET, un total de $L=E$ licencias son creadas por el regulador, cada una de ellas confiere el derecho legal de emitir una unidad de contaminante. Cada firma individual es un competidor perfecto en el mercado de permisos, así el mercado de permisos genera un precio de equilibrio de licencias $p$. Se denota por $l_{i 0}$ la asignación inicial de licencias de la firma $i$, y $l_{i}$ el número de licencias que tiene la firma después de realizar transacciones. En este contexto, la firma $i$ tiene dos variables individuales a su disposición: el nivel de emisiones,

2 La interpretación usual de $c(e)$ es como sigue: sea $e^{0}$ el nivel no restringido de emisiones de una fuente, y sea $b\left(e^{0}\right)$ el nivel máximo de beneficios de la firma en tal contexto. El costo de seleccionar un nivel de emisiones $e<e^{0}$ es $c(e)=b\left(e^{0}\right)-b(e)$. Montgomery (1972) mostró que $c(e)$ es decreciente y convexa cuando la firma es tomadora de precios en mercados de factores y producto final. Debido a que la formulación de $c(e)$ es suficientemente general, la función también posee tales características en diversos contextos no competitivos (ver también Stranlund y Chávez, 2000).

3 La multa es igual a cero cuando la violación es cero pero la multa marginal por violación cero es superior a cero $\left[f(0)=0, f^{\prime}(0)>0\right]$. Para una violación positiva la multa crece a una tasa creciente, esto es, $\left[f^{\prime \prime}(v)>0\right]$.

4 Los costos marginales al nivel del estándar pueden variar a través de las firmas, no sólo porque ellas enfrentan un estándar diferente, sino también porque posiblemente las características específicas de las firmas difieren. 
$e_{i}$, y el número final de licencias $l_{i}$. Una violación de emisiones $v_{i}$ ocurre para la firma $i$ si sus emisiones sobrepasan el número de licencias que posee después de transacciones, o sea $v_{i}=e_{i}-l_{i}>0$.

$\mathrm{El}$ problema de la firma consiste entonces en elegir sus emisiones y su demanda de permisos tratando de minimizar los costos de abatimiento, los ingresos o gastos derivados de la compra o venta de permisos, y la multa esperada. La elección optimal de emisiones requiere que $-c_{i}^{\prime}\left(e_{i}\right)=p$, lo cual define implícitamente $e_{i}(p)$. En cumplimiento, el nivel de emisiones de la firma $i$ es igual a sus demandas de permisos, que es $e_{i}(p)=l_{i}(p)$. La condición de equilibrio en un mercado de permisos de emisión está dada por $\sum_{i}^{N} l_{i}(p)=L=E$; lo cual define implícitamente el precio de equilibrio como una función de los parámetros de costos de abatimiento de las firmas; y del número total de licencias; eso es $p(E)$. Entonces, bajo un SPET, una firma elegirá un nivel de emisiones y una demanda de permisos, que le permita estar en cumplimiento con la regulación cuando se cumpla la siguiente condición (ver Malik, 1990; Stranlund y Dhanda, 1999):

$$
p(L) \leq \pi_{i} f^{\prime}(0)
$$

La ecuación (1b) sugiere que una firma estará en cumplimiento con la regulación cuando la multa marginal esperada no sea menos que el precio equilibrio obtenido en un mercado de permisos competitivo.

\subsection{Estrategias de fiscalización para inducir perfecto cumplimiento ${ }^{5}$}

La fiscalización costo-efectiva requiere lograr el perfecto cumplimiento de cada fuente al mínimo esfuerzo de fiscalización $\left(\pi_{i}\right)$. En el caso de un SPET perfectamente competitivo, Stranlund y Dhanda (1999), y Stranlund y Chávez (2000) han mostrado que si las multas son aplicadas uniformemente, la estrategia de fiscalización costo-efectiva es monitorear cada firma con una única probabilidad, $\pi_{i}=\pi$, eso quiere decir que no es necesario hacer una fiscalización focalizada. Por lo tanto, el mínimo monitoreo requerido para inducir cumplimiento está dado por:

$$
\pi^{\text {min }}=\frac{p(E)}{f^{\prime}(0)}
$$

5 El concepto estrategia de fiscalización se utiliza aquí, siguiendo la literatura existente, para definir la intensidad del esfuerzo de vigilancia medido a través de la probabilidad generada de ser auditado. En este trabajo no se abordan los aspectos prácticos asociados con una implementación del regulador que conlleve a alcanzar los niveles de probabilidad de fiscalización previamente mencionados. 
Al contrario, bajo un estándar de emisión Malik (1992) mostró que la fiscalización costo-efectiva implica un esfuerzo de monitoreo que varía a través de las firmas de acuerdo a sus costos marginales de abatimiento. Sin embargo, para lograr monitoreo perfecto, el regulador debe tener información completa sobre los costos de abatimiento de cada firma regulada. Malik (1992) mostró que bajo supuesto de información completa, el mínimo monitoreo requerido para cada firma está dado por:

$$
\pi_{i}^{\min }\left(s_{i}\right)=\frac{-c_{i}^{\prime}\left(s_{i}\right)}{f^{\prime}(0)}
$$

En un sistema de estándares de emisión con información incompleta, suponemos que, aunque el regulador conoce la distribución de los costos de abatimiento de las firmas, es incapaz de reconocer los costos de abatimiento de cada fuente individual. Este escenario brinda una ventaja de información asimétrica para las firmas que éstas podrían utilizar en su beneficio. Por lo tanto, el regulador necesita anticipar la respuesta estratégica de una firma mediante un diseño de fiscalización apropiado (Chávez et al., 2009). Como la información de que dispone no está individualizada por firma el regulador debe monitorear los diferentes tipos de firmas con la misma probabilidad $(\pi)$, pero de tal manera que se induzca cumplimiento. La fiscalización costo-efectiva en el contexto de un sistema de estándares de emisión con información incompleta requiere un monitoreo mínimo dado por:

$$
\pi^{\min }=\operatorname{Max}\left(\frac{-c_{i}^{\prime}\left(s_{i}\right)}{f^{\prime}(0)}\right), i=1, \ldots, N
$$

Como puede notarse, las estrategias de fiscalización (esfuerzo de monitoreo, $\pi$ ) para inducir perfecto cumplimiento difieren según el tipo de instrumento de control y el supuesto de información considerados. En el caso de un estándar de emisión, la calidad de la información que dispone el regulador influye sobre sus estrategias de fiscalización. Sin embargo, bajo un SPET la información sobre los costos de abatimiento no es relevante para definir las estrategias de fiscalización, debido a que tales estrategias son independientes del tipo de firma.

\subsection{Costos de fiscalización}

El costo de fiscalización $(C F)$ asociado a un instrumento de control determinado depende del esfuerzo de fiscalización (probabilidad de auditar), del costo de hacer una auditoría $(w)$ y del número total de fuentes bajo regulación $(N)$. Dado que el costo de una auditoría y el número de firmas bajo regulación se mantienen constantes para ambos instrumentos bajo estudio, la probabilidad mínima de monitoreo es el elemento que diferencia los costos de fiscalización asociado a cada instrumento.

En el caso de un SPET, los costos de fiscalización para inducir cumplimiento están dados por: 


$$
C F^{S P E T}=w^{*} \frac{p(E)}{f^{\prime}(0)} * N
$$

Considerando un sistema de estándares de emisión con información completa, los costos de fiscalización están dados por la suma de los costos de fiscalización asociados a cada firma:

$$
C F_{1}^{E E}=w^{*} \sum_{i=1}^{N} \frac{-c^{\prime}\left(s_{i}\right)}{f^{\prime}(0)}
$$

Cuando existe información incompleta por parte del regulador y en el caso de un sistema de estándares de emisión, los costos de fiscalización pueden ser expresados como:

$$
C F_{2}^{E E}=w * \operatorname{Max}\left(\frac{-c_{i}^{\prime}\left(s_{i}\right)}{f^{\prime}(0)}\right) * N, i=1, \ldots, N
$$

\subsection{Costos de cumplimiento}

Los costos de cumplimiento (CC) incluyen los costos agregados de abatimiento y costos de fiscalización (Chávez et al., 2009).

El costo de cumplimiento de un SPET está dado por:

$$
C C^{S P E T}=\sum_{i=1}^{N} c_{i}\left(e_{i}(p(E))\right)+w^{*} \frac{p(E)}{f^{\prime}(0)} * N
$$

En un sistema de estándares de emisión con información completa, el costo de cumplimiento está dado por:

$$
C C_{1}^{E E}=\sum_{i=1}^{N} c_{i}\left(e_{i}\left(s_{i}\right)\right)+w * \sum_{i=1}^{N} \frac{-c_{i}^{\prime}\left(s_{i}\right)}{f^{\prime}(0)}
$$

Para el caso de un estándar de emisión con información incompleta, el costo de cumplimiento está expresado como:

$$
C C_{2}^{E E}=\sum_{i=1}^{N} c_{i}\left(e_{i}\left(s_{i}\right)\right)+w^{*} \operatorname{Max}\left(\frac{-c_{i}^{\prime}\left(s_{i}\right)}{f^{\prime}(0)}\right) * N, i=1, \ldots, N
$$

El instrumento costo-efectivo debe minimizar los costos de cumplimiento logrando la misma meta ambiental. Diversos trabajos de investigación han mostrado que los SPETs permiten lograr una meta ambiental al mínimo costo de abatimiento. Sin embargo, esto no es cierto para los costos de fiscalización 
bajo supuesto de información completa. En consecuencia, un SPET podría ser menos o más costoso que un estándar de emisión en términos de costos de cumplimiento si se tiene información completa $\left(C C^{S P E T} \leq C C_{1}^{E E}\right.$ ó $\left.C C^{S P E T} \geq C C_{1}^{E E}\right)$ (Malik (1992)). Como se expondrá más adelante, en nuestro caso de estudio los cálculos realizados evidencian que los costos de cumplimiento de un mecanismo de comando y control en la forma de emisiones son aproximadamente seis veces mayores que los asociados a un sistema de permisos transferibles, en un contexto de información completa, lo que constituye uno de los aportes de nuestro estudio numérico.

En el caso de información incompleta los costos de cumplimiento de un sistema de estándares de emisión no pueden ser más bajos que aquellos de un SPET. Esto se debe a que, por una parte, un SPET permite lograr una meta ambiental al mínimo costo de abatimiento; y por la otra, los costos de fiscalización de estándares de emisión con información incompleta son más altos que los de un SPET $\left(C C_{2}^{E E} \geq C C^{S P E T}\right)$ (Chávez et al., 2009). Este comportamiento teórico para el caso de información incompleta es corroborado con nuestros cálculos. En nuestro caso de estudio se estima que los costos de cumplimiento de un sistema de comando y control resultan ser aproximadamente siete veces mayores que los de un SPET. La cuantificación de la magnitud real de la ventaja de un esquema de regulación ambiental basado en incentivos económicos es otro aporte de nuestro estudio.

Finalmente, hacemos notar que los costos de fiscalización esperados de estándares de emisión bajo información incompleta son más altos que aquellos asociados a estándares bajo información completa $\left(C F_{2}^{E E} \geq C F_{1}^{E E}\right)$. En consecuencia, un sistema de estándares de emisión con información incompleta es más costoso, en términos de costos de cumplimiento, que un sistema de estándares de emisión con información completa $\left(C C_{2}^{E E} \geq C C_{1}^{E E}\right)$, ya que los costos agregados de abatimiento no cambian según la disponibilidad de información del fiscalizador. En nuestros cálculos esta diferencia se observa de la siguiente manera. Los costos de fiscalización del sistema de estándares bajo información incompleta superan en once veces los mismos costos con información completa. Sin embargo, los costos de cumplimiento del mismo sistema con información incompleta sólo aumentan en un ocho por ciento respecto al caso de información completa (ver sección 4). Esta discrepancia en las brechas atribuibles a la falta de información, se explica por las marcadas diferencias de magnitud entre los costos de fiscalización y de abatimiento.

\section{Datos y Calibración del Modelo}

En esta sección se provee una caracterización general de las fuentes consideradas en las simulaciones. Se presenta también, brevemente, la metodología para determinar las formas funcionales de los costos de abatimiento utilizadas. Por último, se describen las simulaciones realizadas. 


\subsection{Caracterización de las fuentes reguladas}

La caracterización descriptiva de las fuentes consideradas fue realizada utilizando la base de datos para el Programa de Compensación de Emisiones (PCE) provista por SESMA, particularmente se consideraron los datos correspondientes al año 2006. Del total de 446 fuentes registradas en el inventario de SESMA para dicho período, 192 fuentes son denominadas fuentes existentes (43\% del total de las fuentes) y 254 fuentes son nuevas (57\% del total de las fuentes) (véase Anexo 1)6. Durante el período 1999-2006, el total de fuentes se redujo en $22 \%$ dado que las fuentes que permanecieron inactivas durante 3 años seguidos fueron borradas de los registros ${ }^{7}$. Con respeto a los procesos, $85 \%$ de las fuentes del año 2006 eran calderas industriales contra 19,9\% en el año 1999. En el año 2006, alrededor de 98,8\% de las fuentes no tenían equipo de abatimiento. Por otra parte, en el mismo período 1999-2006 el porcentaje de fuentes que utilizaban gas natural respecto al total de fuentes creció desde 30,4\% hasta 57,6\%. En las simulaciones, se consideran 413 fuentes (93\%), de las que se dispone de suficiente información sobre los parámetros necesarios.

\subsection{Costos de abatimiento para PM10}

Existen actualmente cinco (5) alternativas (equipos) de control de PM-10 aplicables a las fuentes fijas participando en el Programa de Compensaciones de Emisiones (PCE) en Santiago: Ciclones - Multiciclones - Lavador venturi - Filtro de Mangas - Precipitador electrostático ${ }^{8}$. Corrigiendo las formas funcionales estimadas por O'Ryan y Bravo (2001) por un factor de ajuste, se obtuvieron relaciones funcionales que representan los costos anuales (totales) de la utilización de un determinado equipo de control, de acuerdo al caudal de la fuente considerada ${ }^{9}$. La función de costo total corresponde a la suma de los costos de inversión anualizados, los costos directos de operación y los costos indirectos de operación. Los costos totales son función del caudal de la fuente $\mathrm{Q}\left(\mathrm{m}^{3} \mathrm{~N} / \mathrm{hr}\right)$ y del número promedio de horas de operación (HRS) ${ }^{10}$. El Cuadro 1 muestra las funciones de costos anuales para cada uno de los equipos aplicables a las fuentes fijas en Santiago.

6 En el año 1999, un total 573 fuentes se encontraban participando en el Programa de Compensación de Emisiones (PCE), de las cuales 365 fuentes existentes y 208 fuentes nuevas (Palacios y Chávez, 2002).

7 Contacto personal con Roberto Condori, Subdepartamento Calidad del aire, SESMA (2007).

8 Contacto Personal con Roberto Condori, Subdepartamento Calidad del aire, SESMA (2007).

9 Este factor de ajuste (FA) fue calculado de la siguiente forma: $\mathrm{FA}=\mathrm{IPC}_{2006} / \mathrm{IPC}_{1998}$, donde: $\mathrm{IPC}_{2006}$ : Índice de Precio al Consumidor a Diciembre del 2006, IPC ${ }_{1998}$ : Índice de Precio al Consumidor a Diciembre del 1998.

10 Corresponde al número de horas promedio diario de operación de la fuente, calculada como el número de horas al día de funcionamiento, ponderado por la razón entre el número de días al año de operación de la fuente y los 365 días del año. 


\section{CUADRO 1 \\ FUNCIONES DE COSTOS PARA SANTIAGO \\ (\$ año 2006)}

\begin{tabular}{|lllc|}
\hline \multicolumn{1}{|c}{$\begin{array}{c}\text { Alternativa } \\
\text { de control }\end{array}$} & \multicolumn{1}{c}{$\begin{array}{c}\text { Costo de inversión } \\
\text { anualizado } \$ \text { / año) }\end{array}$} & \multicolumn{1}{c}{$\begin{array}{c}\text { Costos directos de } \\
\text { operación }(\$ / \text { año/hora })\end{array}$} & $\begin{array}{c}\text { Costos indirectos de } \\
\text { operación }(\$ / \text { año })\end{array}$ \\
\hline Ciclones & $77,45 * \mathrm{Q}+80123,4$ & $(0,14 * \mathrm{Q}+143,2) * \mathrm{HRS}$ & 625.783 \\
Multiciclones & $132,25 * \mathrm{Q}+141.385$ & $(0,14 * \mathrm{Q}+143,2) * \mathrm{HRS}$ & 625.783 \\
Lavador Venturi & $144.613^{*} \mathrm{Q}^{0,4037}$ & $(137 * \mathrm{Q}+486.568) * \mathrm{HRS}$ & 7.719 .990 \\
Precipitador Electrostático & $98.845^{*} \mathrm{Q}^{0,6261}$ & $(56,6 * \mathrm{Q}-444.335) * \mathrm{HRS}$ & 5.387 .894 \\
Filtro de Mangas & $4.423 * \mathrm{Q}^{0,7449}$ & $(28,6 * \mathrm{Q}+480.423) * \mathrm{HRS}$ & 8.587 .433 \\
\hline
\end{tabular}

Fuente: Elaboración propia basada en O’Ryan y Bravo (2001), pág. 274.

\subsection{Simulaciones: SPET versus Estándares de Emisión}

A continuación se presenta la metodología utilizada en las simulaciones para obtener los costos agregados de cumplimiento.

\subsubsection{Costos de abatimiento: SPET}

El modelo implementado proviene de Bravo (2000), el cual se define de la siguiente manera:

$$
\operatorname{Min} \sum_{i=1}^{413} \sum_{j=1}^{6} C T_{i, j} X_{i, j}
$$

En el que $C T_{i, j}=$ costo anual de aplicación de la tecnología $j$ a la fuente $i$ en $\mathrm{M} \$$ /año, y $X_{i, j}=$ variable de decisión que determina el tipo de tecnología que se aplica a la fuente tipo $i$. Toma el valor 1 si la tecnología $j$ se aplica a la fuente $i$; y cero en otro caso.

Las restricciones que se imponen son las siguientes:

$$
\begin{gathered}
\sum_{i=1}^{413} \sum_{j=1}^{6} E_{i}\left(1-E F_{i, j}\right) X_{i, j} \leq E \\
\sum_{j=1}^{6} X_{i, j}=1
\end{gathered}
$$

Donde $E_{i}=$ emisiones totales de la fuente $i$ en $\mathrm{kg} / \mathrm{año}, \mathrm{y} E F_{i, j}=$ eficiencia de reducción de emisiones de la tecnología $j$ aplicada a la fuente $i$ y $E=$ meta total de emisiones deseada por el regulador en $\mathrm{kg} / \mathrm{año}$. La restricción (6) expone que la distribución resultante de equipos de control a través de las firmas debe 
ser tal que las emisiones agregadas finales sean no superiores al nivel de emisiones prefijado por el regulador. La restricción (7) expone que las fuentes deben escoger sólo una tecnología de control.

Este modelo se resolvió utilizando el software CPLEX, el cual permite optimizar modelos con variables enteras. Considerando que bajo un sistema de permisos de emisiones transferibles se logra cumplir la meta ambiental al mínimo costo agregado de abatimiento, la solución del problema definido por (5), (6) y (7) entrega el costo agregado de abatimiento para la política de control SPET.

\subsubsection{Costos de abatimiento: Estándares de Emisión}

Para obtener el costo agregado de abatimiento, se utilizó una planilla Excel, la cual simula la decisión individual de cada fuente. Primeramente el nivel de reducción porcentual prefijado por el regulador se exige como la reducción mínima para cada fuente. Este proceso fija de manera automática el estándar de emisión de cada fuente $s_{i}$. En la simulación cada fuente elige entonces del conjunto de opciones de control que cumplen con su estándar de emisión, aquella que minimiza su costo de control. Luego, basado en la elección individual de las fuentes, se calcula el costo agregado de abatimiento y el nivel agregado de emisiones finales.

\subsubsection{Sistema de fiscalización}

Como ya mencionamos en la sección 2, la probabilidad mínima de monitoreo es el elemento que diferencia los costos de fiscalización asociados a cada instrumento de regulación.

Se supone que la multa por unidad de violación $(v)$ es una función lineal del precio del mercado bajo el SPET. En tal caso la multa tiene la siguiente forma:

$$
f(v)=\alpha^{*} p^{*} v
$$

En el que $\alpha$ es un factor de sanción, con $\alpha>1$ y $p$ es el precio de mercado por permiso a emitir una unidad de contaminante ${ }^{11}$. Este diseño de sanción dependiente del precio del permiso ha sido sugerido por ejemplo en Stranlund y Chávez (2000). Las ventajas de este mecanismo son discutidas en la referencia citada y el nivel de $\alpha$ está sujeto, naturalmente, a cierto grado de flexibilidad por parte del regulador.

Derivando en (8) y reemplazando en (3a) se obtiene la expresión utilizada para calcular el costo de fiscalización asociado con un SPET.

$$
C F^{S P E T}=w^{*} \frac{1}{\alpha} * N
$$

\footnotetext{
11 El precio del permiso fue calculado como la reducción en los costos agregados de abatimiento si la meta ambiental fuera incrementada de una unidad adicional.
} 
En el caso de un sistema de estándares de emisión el cálculo de la probabilidad mínima de monitoreo requiere el uso de los costos marginales de abatimiento (ver (2b) y (2c)). Sin embargo, debido a que las funciones de costos de abatimiento evaluadas dependen del caudal de la fuente y del promedio de horas de operación (variables discretas), éstas tienen una relación funcional del tipo constante a trozos y, por ende, no diferenciable. Consecuentemente, no es posible encontrar el costo marginal de abatimiento (beneficio marginal de violar) y tampoco calcular la multa marginal esperada (costo marginal esperado de violar) de la manera esbozada en el modelo teórico de la sección 2. Entonces, se optó por calcular la probabilidad de monitoreo de cada fuente de acuerdo al procedimiento descrito en el Anexo 2 y que implica una maximización del tipo,

$$
\pi_{i}=\operatorname{Max}\left(\pi_{i, j}\right)
$$

El costo agregado de fiscalización del sistema de estándares de emisión bajo el supuesto de información completa está dado por:

$$
C F_{1}^{E E}=w^{*}\left[\pi_{1}+\pi_{2}+\ldots+\pi_{N}\right]
$$

Con información incompleta, el costo agregado de fiscalización del sistema de estándares de emisión se expresa como sigue:

$$
C F_{2}^{E E}=w^{*} \operatorname{Max}\left(\pi_{i}\right) * N, i=1, \ldots, N
$$

El Cuadro 2 resume los parámetros utilizados en el cálculo de los costos de fiscalización considerados.

\section{CUADRO 2}

PARÁMETROS DE COSTOS DE FISCALIZACIÓN

\begin{tabular}{|lc|}
\hline Parámetros & Valores \\
\hline Factor de sanción $(\alpha)$ & 4,9 \\
Precio $^{\mathrm{a}}$ & 0,72 \\
Multa por unidad de violación $^{\mathrm{b}}$ & 3,5 \\
Costo de auditoría $^{\mathrm{b}}$ & 0,5 \\
\hline
\end{tabular}

Fuente: Elaboración propia.

a Valor expresado en millones de pesos por kilogramo de PM10.

b Valor expresado en millones de pesos. 
El costo de una auditoría se fijó siguiendo la literatura (Villegas y Chávez, 2004) en un monto aproximado de mil dólares. La multa por violación se determina como el producto del factor de sanción y el precio. Como mencionamos anteriormente, el factor de sanción $\alpha$ es ajustable por el regulador y fue fijado para este estudio de la siguiente manera. Se sustituyó en la fórmula (2c) la definición de multa marginal proveniente de (8). Para que la probabilidad calculada en (2c) exista se necesita que se cumpla la siguiente relación:

$$
\alpha \geq \operatorname{Max}\left(\frac{-c_{i}^{\prime}\left(s_{i}\right)}{p}\right)
$$

Tomando los costos marginales aproximados mediante la metodología explicada en el Anexo 2, se calculó el mínimo $\alpha$ posible que cumpliera la anterior condición. Esta construcción garantiza que la probabilidad mínima de monitoreo que se obtiene derivando (8) y reemplazando en (2a) induzca el perfecto cumplimiento de las firmas en cada uno de los instrumentos bajo estudio.

\section{Resultados}

En esta sección se presentan los resultados de la simulación numérica respecto a la implementación de las políticas de control SPET y estándares de emisión. Para ambos instrumentos se presentan los costos agregados de abatimiento $(C A)$, los costos agregados de fiscalización $(C F)$ y los costos agregados de cumplimiento $(C C)$ obtenidos mediante la simulación numérica. Además, se presentan los resultados derivados del análisis de sensibilidad.

\subsection{Antecedentes}

La naturaleza de las decisiones en este modelo es discreta ya que se basa en cinco tipos de equipos de control, cada uno con un valor prefijado de la eficiencia de reducción. En consecuencia, los niveles de reducción alcanzables, tanto para cada firma como agregados, varían abruptamente mediante saltos. Ese comportamiento provoca que los niveles de emisión para las metas elegidas no sean exactamente obtenidos, sino que haya una tendencia al exceso de reducción de emisiones. La meta considerada es un $40 \%$ de reducción de emisiones con respecto a la situación inicial, que se toma como la ausencia de equipos de control en todas las fuentes. El nivel agregado de las emisiones de las 413 fuentes consideradas asciende en la situación inicial a un total de $1.995 \mathrm{~kg} /$ día equivalente a 728,3 ton/año. La meta ambiente corresponde entonces a lograr un nivel agregado de emisiones inferior a 1.197,2 kg/día (436,9 ton/año). Los resultados de las simulaciones en cuanto a los costos agregados se resumen en el Cuadro 3. 
CUADRO 3

COSTOS AGREGADOS DE CUMPLIMIENTO, SEGÚN POLÍTICA DE CONTROL

(Millones de pesos por año)

\begin{tabular}{|lccc|}
\hline Política de control & $\begin{array}{c}\text { Costo agregado } \\
\text { de abatimiento }\end{array}$ & $\begin{array}{c}\text { Costo agregado } \\
\text { de fiscalización }\end{array}$ & $\begin{array}{c}\text { Costo agregado } \\
\text { de cumplimiento }\end{array}$ \\
\hline EE con IC & 512,3 & 3,9 & 516,2 \\
EE con II & 512,3 & 43,3 & 555,6 \\
SPET & 43,2 & 41,3 & 84,5 \\
\hline
\end{tabular}

Fuente: Elaboración propia.

\subsection{Costos agregados de abatimiento según Sistema de Regulación}

Los resultados obtenidos para la implementación de un SPET revelan que lograr la meta ambiental de 436,9 ton/año de MP tiene un costo agregado de abatimiento de 43,2 millones de pesos al año (91 mil dólares), con un precio de permiso de 0,72 millones de pesos por kilogramo (1.512 dólares $/ \mathrm{kg}$ ) de material particulado emitido ${ }^{12}$. En relación al estándar de emisión, los resultados indican que alcanzar 436,9 ton/año de MP tiene un costo agregado de abatimiento de 512,3 millones de pesos al año (1.076.000 dólares). El costo agregado de abatimiento no cambia con la disponibilidad de información en el caso del estándar de emisión.

Estos resultados indican que el SPET permite lograr la meta ambiental al mínimo costo agregado de abatimiento, en forma consistente con las predicciones teóricas. Cabe notar que el costo agregado de abatimiento resultante del sistema SPET es 8,4\% del obtenido en estándares de emisiones. Esta importante diferencia observada se explica por la flexibilidad que brinda este instrumento a las fuentes reguladas. En la simulación del caso de estudio, sólo seis fuentes (las más grandes) escogen algún tipo de equipo de control en el logro de la meta prefijada. En este contexto, debe considerarse que las funciones de costo de abatimiento estimadas por O'Ryan y Bravo (2001) permiten a dichas fuentes (caudales grandes) obtener niveles de costos de abatimiento menores que fuentes pequeñas (caudales pequeños) según el equipo de control considerado, lo cual se traduce en una reducción del costo agregado de abatimiento de 461,1 millones de pesos al año (ahorro en costos del 91,6\%). Por otra parte, al utilizar una política de estándares de emisión, todas las firmas deben escoger un equipo de control para cumplir con su meta individual, no existiendo la posibilidad de transferir sus obligaciones ambientales a otras empresas más eficientes, lo cual incrementa los costos agregados de abatimiento asociados.

\subsection{Costos de fiscalización}

Los costos agregados de fiscalización dependen principalmente de la política ambiental considerada y además varían dentro una misma política según la

12 Los cálculos consideran 1 dólar equivale a 476 pesos. 
información disponible. Con respecto a la regulación basada en un SPET, los resultados indican que la probabilidad de monitoreo requerida para inducir perfecto cumplimiento tiene un valor de 0,2 , este valor se obtiene como $\pi^{\mathrm{min}}=1 / \alpha$, a partir de las ecuaciones (2a) y (8) y la información provista en el Cuadro 2. Esta probabilidad representa un costo esperado de fiscalización para el regulador de 41,3 millones de pesos al año (87 mil dólares), según la ecuación (9).

En relación con la política de comando y control, el costo esperado de fiscalización de un sistema de estándares de emisión bajo supuesto de información completa asciende a 3,9 millones de pesos al año (8 mil dólares), que se calcula según la ecuación (11a). Un sistema de estándares de emisión bajo supuesto de información incompleta tiene asociado un costo esperado de inducir cumplimiento de 43,3 millones de pesos al año (90 mil dólares), calculado a su vez, de acuerdo a la expresión (11b). Luego, se desprende que asimetrías de información respecto de los costos de abatimiento de las firmas por parte de la autoridad regulatoria, cuando se consideran estándares de emisión, pueden incrementar los costos agregados de fiscalización de manera significativa, lo cual en este caso de estudio, dado los parámetros utilizados, se traduce en 39,4 millones de pesos por año. Este resultado empírico es de suma relevancia en la implementación práctica de estándares de emisión, en particular en países en vías de desarrollo, ya que el no considerar los costos de fiscalización en el diseño de políticas ambientales puede implicar fallas en la implementación de este tipo de programas por la falta de presupuesto para fiscalizar su correcto funcionamiento.

Dado que el costo de una auditoría es invariante entre sistemas regulatorios y no depende de la disponibilidad de información, las diferencias en los costos de fiscalización asociados a inducir perfecto cumplimiento se deben a diferencias en el número esperado de firmas que deben ser auditadas, lo cual a su vez varía entre sistemas de regulación y disponibilidad de información a través de la probabilidad mínima necesaria para inducir cumplimiento. En el caso de la regulación basada en un sistema de estándares de emisión con información incompleta, la probabilidad monitoreo requerida es la máxima entre las que se obtienen para todas las firmas reguladas (ver expresión (11b)). La existencia de una firma con una probabilidad necesaria mucho más grande que las demás explica la diferencia en magnitud resultante de aplicar las expresiones (11b) y (11a).

En el caso de un SPET, el costo de fiscalización se acerca en magnitud a aquel obtenido en el caso de un sistema de estándares con información incompleta. El rol principal para explicar tal diferencia lo tiene el cálculo de la probabilidad mínima de monitoreo de acuerdo a las expresiones (2a) y (2c). La manera en que se calculó el numerador en ambas expresiones explica la similitud entre los resultados (nótese que la expresión (2c) incluye una derivada que no es posible calcular en nuestro contexto). Especialmente, en el caso del precio existe probablemente una sobrestimación de éste asociada a que se imputa todo el valor de una adopción de tecnología de abatimiento al cambio en una unidad de la meta ambiental (ver sección 3.3.3).

El análisis de los resultados anteriores indica que inducir perfecto cumplimiento en un sistema de regulación basado en estándares de emisión con información completa es más barato que en el caso de información incompleta o de un SPET. Sin embargo, fiscalizar un SPET es más barato que un estándar 
de emisión con información incompleta. Como mencionamos anteriormente en la sección 2 este último resultado es teóricamente esperable.

Del Cuadro 3 también se concluye que los costos de fiscalización, respecto de los costos agregados de cumplimiento, cuando se consideran estándares de emisión, no son tan relevantes si se cuenta con información completa de los costos de abatimiento reales de las distintas firmas. De hecho en este caso de estudio, cuando existe información completa por parte de la autoridad regulatoria, los costos de fiscalización representan sólo un $0,75 \%$ de los costos agregados de cumplimiento. En cambio, cuando existe información incompleta esta razón aumenta a un 7,7\%. Dada esta línea de análisis se puede concluir que la presencia de información incompleta aumenta los costos agregados de cumplimiento en un $7,6 \%$.

En términos de un SPET, del Cuadro 3 se puede concluir que los costos agregados de fiscalización son muy relevantes a la hora de considerar los costos agregados de cumplimiento de este tipo de regulación ambiental. En el caso bajo estudio, dado los parámetros utilizados, los costos de fiscalización representan un $48,9 \%$ de los costos agregados de cumplimiento. En consecuencia, el no considerar los costos de fiscalización en el diseño de un SPET puede llevar a subestimar los costos totales de implementación de esta política en casi la mitad de su costo real. De hecho del Cuadro 3 resulta claro que en términos de un SPET los costos agregados de fiscalización son tan relevantes como los costos agregados de abatimiento (43,2 versus 41,3 millones de pesos al año).

\subsection{Costos de cumplimiento}

Se puede observar que para reducir los niveles de emisiones agregadas desde 728,3 ton/año a 436,9 ton/año, el costo agregado de cumplimiento asociado a un sistema de estándares de emisión con información completa alcanza a 516,2 millones de pesos al año (1.084.000 dólares). En relación con el estándar de emisión con información incompleta, su costo agregado de cumplimiento es 555,6 millones de pesos al año (1.166.000 dólares). Con respecto al SPET, su costo agregado de cumplimiento alcanza a 84,5 millones de pesos al año (178 mil dólares).

De los resultados expuestos en el Cuadro 3 también se desprende que al considerarse los costos agregados de fiscalización, el potencial ahorro en costos obtenido por el uso de instrumentos económicos para el control de la contaminación, en lugar de instrumentos de comando y control, se ve reducido. En particular, en este caso el ahorro obtenido mediante la implementación de SPET en lugar de estándares de emisión cambia desde un 91,6\% cuando no se consideran los costos de fiscalización, a un ahorro de un $83,6 \%$ y $84,8 \%$ para los casos de información completa e incompleta, respectivamente.

Por lo tanto, se puede argumentar que el considerar los costos de fiscalización reduce el ahorro esperado derivado de instaurar un SPET a partir de un sistema de estándares de emisión. Además, el ahorro porcentual resultante permanece similar a través de ambos escenarios de información considerados.

El análisis de los resultados para los costos de cumplimiento asociados con ambas políticas indica que el instrumento de mercado es más barato en términos de costos de cumplimiento (costos de abatimiento más costos de fiscalización) que el instrumento de comando y control. En cuanto al caso de un estándar de 
emisión, el costo de cumplimiento con información incompleta es mayor que si la información estuviera disponible. Naturalmente, esta última relación es una evidencia numérica de los resultados teóricos presentados en la sección 2.

La diferencia de magnitud observada entre un SPET y un sistema de estándares de emisión puede asociarse mayormente a las diferencias en los costos de abatimiento que han sido previamente abordadas. Esto es consecuencia de que, en este caso de estudio, la magnitud de los costos de fiscalización no alcanza a influir sobre las diferencias entre los costos agregados de abatimiento.

\subsection{Análisis de sensibilidad}

En esta sección se presentan los resultados de las simulaciones del análisis de sensibilidad. Dicho análisis permite evaluar el impacto en los costos agregados de cumplimiento para ambas políticas ambientales en los escenarios de información completa e incompleta, ante cambios en parámetros relevantes para el estudio de los costos de abatimiento, de fiscalización y de cumplimiento. Los escenarios simulados son los siguientes: (1) cambios en meta ambiental, (2) cambios en costos de abatimiento, (3) cambios en la sanción por incumplimiento, (4) cambio en número de fuentes reguladas.

\section{Cambios en meta ambiental}

Para el caso de información completa se estudian los efectos de los cambios en meta ambiental sobre la selección de tecnología de control. Las variaciones de la meta ambiental pueden estar asociadas a cambios de política o ajustes dinámicos de los objetivos del regulador. La selección de tecnología de control resultante para cada meta ambiental afecta el costo agregado de abatimiento y el nivel de precio del permiso de emisión. El cambio en el precio afecta la multa esperada, lo que a su vez influye sobre la probabilidad de monitoreo y por lo tanto afecta tanto los costos agregados de fiscalización como los costos agregados de cumplimiento. Dado el aspecto discreto de la eficiencia de los equipos, las metas seleccionadas para el análisis de sensibilidad corresponden a una reducción de $24 \%$ y $63 \%$ de los niveles de emisión.

Analizando el efecto de una meta ambiental menos estricta con un SPET, se observa que el costo agregado de cumplimiento disminuye más de 4 veces y el precio disminuye en $30 \%$ respecto a la situación de base. Sin embargo, al incrementar la exigencia de la meta ambiental, el costo agregado de cumplimiento aumenta más de 2 veces y el precio del permiso disminuye en $51 \%$. La disminución en el nivel de precio del permiso de emisión con una meta más ambiciosa es un fenómeno inesperado. Este resultado está determinado por el diseño de las formas funcionales de costos de abatimiento que permite reducir un porcentaje más alto de emisión con un costo de abatimiento asociado menor según el equipo considerado y el caudal de la fuente. Ello disminuye la multa esperada e incrementa el incentivo a transgredir. Lo último implica un aumento en el esfuerzo de fiscalización de parte del regulador y redunda en un aumento de los costos esperados de fiscalización. Eso explica que el costo agregado de fiscalización del SPET ha aumentado más de 2 veces respecto a la situación de base. 
En el caso de un estándar de emisión, al reducir la exigencia ambiental, el costo agregado de fiscalización disminuye en $37 \%$ y el costo agregado de cumplimiento disminuye en $2 \%$ en relación a la situación inicial. En contraste, para lograr una meta ambiental más ambiciosa, el costo de fiscalización aumenta en $302 \%$ y el costo agregado de cumplimiento se incrementa más de 2 veces con respecto a la situación inicial. El aumento en los costos de fiscalización al incrementar la exigencia en la meta ambiental es sorprendente. Lo anterior se produce debido a que el precio del permiso de emisión disminuye ante metas ambientales más exigentes; la multa esperada ha disminuido, por lo tanto ha aumentado la probabilidad de monitoreo individual para cada fuente de parte del regulador (fiscalización focalizada). En consecuencia, se aumentan los costos agregados de fiscalización del estándar de emisión. El Gráfico 1 presenta los costos agregados de cumplimiento para distintos niveles de exigencia de la meta ambiental considerando ambas políticas de control.

\section{GRÁFICO 1 \\ COSTOS AGREGADOS DE CUMPLIMIENTO \\ (Millones de pesos)}

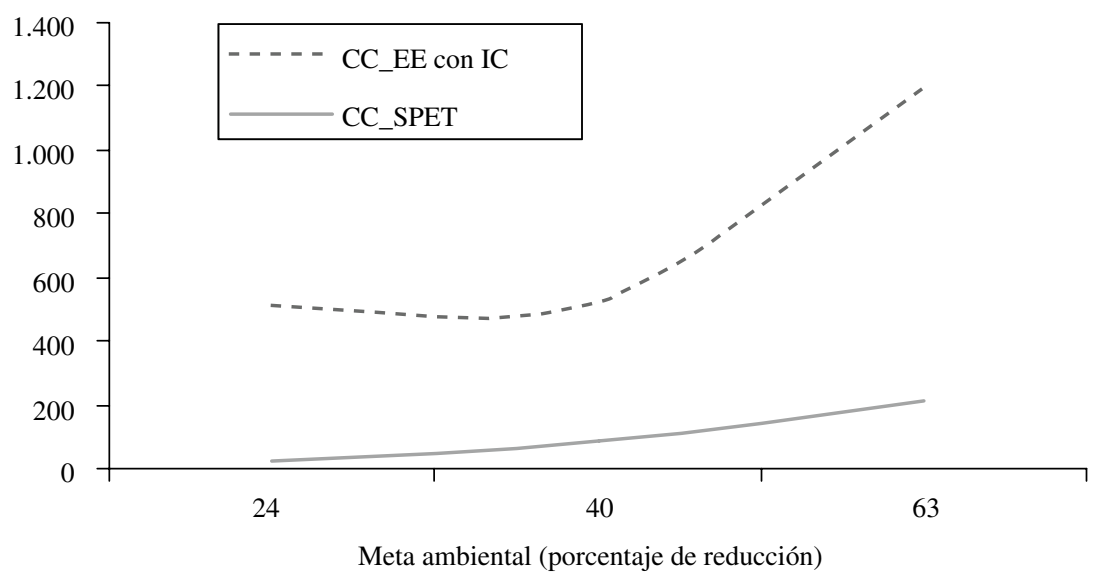

Fuente: Elaboración propia.

\section{(2) Cambios en costos de abatimiento}

El análisis de sensibilidad sobre los costos de abatimiento consiste en aumentos (disminuciones) de $25 \%$ de los valores de las funciones de costo de abatimiento de cada fuente. La motivación para este análisis de sensibilidad es la incertidumbre inherente a los valores reales de las funciones de costo. No se alteran en este contexto las opciones tecnológicas de control.

Los resultados obtenidos de las simulaciones de cambios en las funciones de costos de abatimiento sugieren que, como es obvio, al aumentar (reducir) los costos de los equipos de control en $25 \%$, los costos agregados de abatimiento 
aumentan (reducen) en igual magnitud para ambas políticas. Sin embargo, los efectos se reflejan diferentes en los costos agregados de cumplimiento. Así, los costos agregados de cumplimiento de la política de comando y control aumentan (reducen) en $24 \%$ ante variaciones en costos de abatimiento. En relación a la política de mercado, los costos agregados de cumplimiento aumentan (reducen) en $13 \%$ ante cambios en costos de abatimiento. Los resultados indican entonces que los costos agregados de cumplimiento de la política de comando y control son más sensibles que los de la política de mercado ante cambios en costos de los equipos de control. Lo anterior se debe a la diferencia en la participación relativa de los costos agregados de fiscalización en los costos agregados de cumplimiento entre medidas regulatorias.

\section{Cambios en la sanción por incumplimiento}

Los cambios en la sanción por incumplimiento simulados corresponden en aumentos (disminuciones) de $50 \%$ en el factor de sanción con respecto a la situación de base. Una utilidad de este ejercicio es la cuantificación que se obtiene de las variaciones en los costos de fiscalización ante cambios en el nivel de sanción por unidad de violación. No se alteran los costos agregados de abatimiento porque el factor de sanción no depende de las elecciones de equipos por parte de las fuentes. Sin embargo, debido a que cambios en el factor de sanción implican variaciones en la multa esperada por incumplimiento, por lo tanto los costos agregados de fiscalización son afectados.

Al aumentar el factor de sanción en $50 \%$, los resultados indican que los costos agregados de fiscalización disminuyen para ambas políticas. Los CF caen en $35 \%$ en el caso del SPET y en $33 \%$ en el caso del estándar de emisión. Lo anterior se explica por el hecho que el aumento en el factor de sanción incrementa la multa esperada, la que aumenta el incentivo al cumplimiento. Por otro lado, la probabilidad de monitoreo disminuye, reduciendo los costos agregados de fiscalización. Cuando se disminuye el factor de sanción el efecto es inverso pero en magnitudes diferentes. Los CF aumentan en $100 \%$ para la política de mercado y en $98 \%$ en el caso de la política normativa de emisión.

Con respecto a los costos agregados de cumplimiento, los aumentos (disminuciones) en el factor de sanción reducen (aumentan) los CC para ambas políticas. Cuando se aumenta (disminuye) el factor de sanción para la política de comando y control, el CC disminuye (aumenta) en magnitudes menos a 1\%. Sin embargo, el CC para la política de mercado disminuye en $17 \%$ ante aumento en el factor de sanción y aumenta en $49 \%$ ante disminución en el factor de sanción. Los resultados anteriores muestran que los CC de la política de mercado son más sensibles ante disminuciones en factor de sanción.

\section{Cambio en número de fuentes reguladas}

En este escenario, se considera un total de 250 fuentes $(60 \%$ del total de la situación inicial), cuyo nivel de emisiones iniciales individual sea al menos igual a $70 \mathrm{~kg} / \mathrm{año.} \mathrm{El} \mathrm{nivel} \mathrm{agregado} \mathrm{de} \mathrm{emisiones} \mathrm{inicial} \mathrm{del} \mathrm{total} \mathrm{de} \mathrm{fuentes}$ consideradas corresponde a $1.979 \mathrm{~kg}$ /día (722 ton/año), lo que representa $99 \%$ de las emisiones totales de la situación de base. Se mantiene la misma meta ambiental y el mismo factor de sanción de la situación de base. La meta am- 
biental equivale a 433,4 ton/año. Se esperan cambios en los CA consecuencia del cambio en la selección de equipo, en los CF consecuencia del cambio en el precio de permiso de emisión y en el número de fuentes reguladas. Ellos implican cambios en los CC para ambas políticas.

Los resultados obtenidos de la simulación de cambio en número de fuentes reguladas indican disminuciones considerables en los CA, los CF y los CC para ambas políticas. Por ejemplo, las simulaciones indican altos niveles de ahorro en los CF para ambas políticas. Los costos agregados de fiscalización se reducen en $40 \%$ para la política de mercado y en $82 \%$ para el caso de política normativa de emisión. El análisis sugiere que los $\mathrm{CF}$ de la política de comando y control son más sensibles a disminuciones en el número de fuentes reguladas. Lo anterior se debe a que menor número de fuentes implica menores esfuerzos de monitoreo dado que el regulador responde con una estrategia de fiscalización focalizada. Con respecto a los CC, se observa una disminución en magnitudes casi igual para ambas políticas ante una disminución de $40 \%$ en el número de fuentes reguladas.

\section{Conclusiones}

En este trabajo, se ha evaluado numéricamente la propiedad de costoefectividad de un sistema de permisos de emisión transferibles y un sistema de estándares de emisión individuales. Nuestro análisis incorpora, por una parte, los costos de fiscalización necesarios para inducir perfecto cumplimiento y, por la otra, la diferenciación de los casos con información completa e incompleta. Las simulaciones se desarrollaron en el contexto de las fuentes participando en el Programa de Compensación de Emisiones (PCE) de Santiago de Chile. Finalmente, los resultados obtenidos fueron expuestos a un estudio de sensibilidad respecto a los parámetros de las simulaciones.

Los resultados numéricos obtenidos corroboran que la política de incentivos económicos permite mejorar la calidad del aire al mínimo costo de abatimiento. Sin embargo, este artículo provee un ejemplo en el cual la política de mercado, bajo supuesto de información completa, no es capaz de lograr mejoras en la calidad del aire al mínimo costo de fiscalización. También se obtiene una comprobación numérica de un resultado reciente, que establece que los costos de inducir perfecto cumplimiento en un SPET nunca exceden al caso de una política de control directo de emisiones bajo supuesto de información incompleta.

En el caso de la política de comando y control, se ha cuantificado la ventaja teórica en cuanto a los costos de cumplimiento que se genera debido a la existencia de información completa. Lo anterior revela la importancia de la información en el diseño de una política de comando y control. Finalmente, los resultados obtenidos indican que en términos de costos de cumplimiento, la política basada en un SPET es más barata que la política de comando y control. Este resultado es conocido en el caso de información incompleta, pero no demostrado en el caso de información completa.

Este trabajo empírico expande los resultados teóricos presentados en la literatura previa relacionada, presentando una cuantificación más detallada de los potenciales efectos de incluir los costos agregados de fiscalización en el análisis. Primero, una conclusión importante de este trabajo es que asimetrías 
de información respecto de los costos de abatimiento de las firmas por parte de la autoridad reguladora, cuando se consideran estándares de emisión, pueden incrementar los costos agregados de fiscalización significativamente. Este resultado empírico es de suma relevancia en la implementación práctica de estándares de emisión, en particular en países en vías de desarrollo, ya que el no considerar los costos de fiscalización en el diseño de políticas ambientales puede implicar fallas en la implementación de este tipo de programas por la falta de presupuesto para fiscalizar su correcto funcionamiento.

Segundo, de este trabajo también se puede concluir que los costos de fiscalización, respecto de los costos agregados de cumplimiento, cuando se consideran estándares de emisión, no son tan relevantes si se cuenta con información adecuada (completa) de los costos de abatimiento reales de las distintas firmas.

Tercero, en términos de un SPET, concluimos que los costos agregados de fiscalización son relevantes al considerar los costos agregados de cumplimiento. En el caso bajo estudio, dado los parámetros utilizados, los costos de fiscalización representan un 48,9\% de los costos agregados de cumplimiento. Entonces, el no considerar los costos de fiscalización en el diseño de un SPET puede llevar a subestimar los costos totales de implementación de esta política en casi la mitad de su costo real. De hecho, del caso de estudio analizado resulta claro que en términos de un SPET los costos agregados de fiscalización son tan relevantes como los costos agregados de abatimiento.

Cuarto, de los resultados expuestos en este artículo se desprende que al considerarse los costos agregados de fiscalización, el potencial ahorro en costos obtenido por el uso de instrumentos económicos para el control de la contaminación, en lugar de instrumentos de comando y control, se ve reducido. En particular, el considerar los costos de fiscalización reduce el ahorro esperado derivado de instaurar un SPET a partir de un sistema de estándares de emisión.

Los resultados obtenidos del análisis de sensibilidad revelaron que cambios en los parámetros utilizados en las simulaciones no afectan significativamente las conclusiones principales de este artículo.

La lección empírica de nuestro ejercicio puede resumirse en las siguientes observaciones. En primer lugar, la información disponible al regulador es vital para la propiedad de costo-efectividad respecto a los costos de fiscalización. En segundo lugar, debido a las diferencias de magnitud observadas, el orden de costo-efectividad de las políticas respecto a los costos de abatimiento no se altera al adicionar los costos de fiscalización, que pueden obedecer a un orden diferente en relación a este otro costo. Por último, en tercer, lugar el sistema de permisos de emisión transferibles es más costo-efectivo en cuanto a costos de cumplimiento, siendo este resultado robusto respecto a variaciones en las hipótesis de cálculo.

Finalmente, cabe destacar que más allá de la inclusión de costos de fiscalización en el análisis, de la consideración de distintos niveles de información respecto a las firmas reguladas y de la potencial variación que pudieran tener los parámetros considerados de este trabajo empírico, resulta claro que la ventaja de instrumentos económicos para el control de la contaminación sobre instrumentos de comando y control en términos de eficiencia económica es significativa y robusta. Este resultado ayuda a despejar y a aclarar la ambigüedad teórica expuesta en la literatura económica reciente en particular en relación al caso de información completa. 


\section{REFERENCIAS}

Arimura, T. (2002). "An Empirical Study of the $\mathrm{SO}_{2}$ Allowance Market: Effects of PUC Regulations". Journal of Environmental Economics and Management, Vol. 44, Issue 2: 271-289.

Bravo, R. (2000). "Proposición y Evaluación de Instrumentos de incentivo Económico para Mejorar la Calidad del Aire en Santiago: Aplicación al Caso de Fuentes Fijas". Tesis de Ingeniero Civil, Facultad de Ciencias Físicas y Matemáticas, Universidad de Chile, inédito.

Burtraw, D. 1996. "The $\mathrm{SO}_{2}$ Emissions Trading Program: Cost Saving without Allowance Trades". Contemporary Economic Policy, Vol. 14, Issue 2: 79-94.

Burtraw, D. 1999. "Cost Savings, market performance and economic benefits of the US Acid Rain Program". In Pollution for Sale: Emissions and Joint Implementation. S. Sorrell and J. Skea Cheltenhanm, UK, Edaward Elgar Publishing Limited: 43-62.

Chávez, C.; Villena M. y Stranlund, J. (2009). "The Choice of Instruments to control Pollution under Costly Enforcement and Imperfect Information". Aceptado, por aparecer en Journal of Applied Economics.

Hahn, R. and Axtell, R. (1995), Reevaluating the Relationship between Transferable Property Rights and Command and Control Regulations, Journal of Regulatory Economics 8: 125-148.

Heyes, A. (2000). "Implementing Environmental Regulation: Enforcement and Compliance". Journal of Regulatory Economics 17 (2): 107-129.

McLean, B. (1997). Evolution of Marketable Permits: The US Experience with Sulfur Dioxide Allowance Trading, International Journal of Environment and Pollution 8 (1-2): 19-36.

Malik, A. (1990). "Markets for Pollution Control when Firms are Noncompliant". Journal of Environmental Economics and Management 18: 97-106.

Malik, A. (1992). "Enforcement Cost and the Choice of Policy Instruments for Controlling Pollution". Economic Inquire, Vol. 30; Issue 4; 714-721.

Montgomery, W. D. (1972). "Markets in Licenses and Efficient Pollution Control Programs". Journal of Economic Theory, 395-418.

O'Ryan, R. (1996). "Cost-Effective Policies to Improve Urban Air Quality in Santiago, Chile". Journal of Environmental Economics and Management, $\mathrm{N}^{\mathrm{o}} 31 ; 302-313$.

O’Ryan, R. y Bravo, R. (2001). "Permisos Transables Frente a la Introducción de un Combustible Limpio: Estudio de caso para PM-10 y NOx en Santiago, Chile”. Estudios de Economía, Vol. 28 (2): 267-291.

Palacios, M. y Chávez, C. (2002). "Programa de Compensación de Emisiones. Evaluación del Diseño de Fiscalización y su Cumplimiento". Estudios Públicos, $\mathrm{N}^{\circ} 88$ 97-126.

Schmalensee, R.; Joskow, P.; Ellerman, A. D.; Montero, J. P. y Bailey, E. (1998). An Interim Evaluation of Sulfur Dioxide Emissions Trading, The Journal of Economic Perspectives 12 (3): 53-68. 
Stavins, R. (1998). What Can We Learn from the Grand Policy Experiment? Lessons from the $\mathrm{SO}_{2}$ Allowance Trading, The Journal of Economic Perspectives 12 (3): 69-88.

Stranlund, J. y Chávez, C. (2000). "Effective Enforcement of a Transferable Emission Permit System with a Self Reporting Requirement". Journal of Regulatory Economics, vol. 18: 113-131.

Stranlund, J. y Dhanda, K. (1999). "Endogenous Monitoring and Enforcement of a Transferable Emissions Permit System". Journal of Environmental Economics and Management, 38 (3): 267-282.

Villena, M. y Villena, M. (1998). "Analysing Economic Strategies for Air Pollution Control in Santiago, Chile". Environmental Series $\mathrm{N}^{\circ}$ 8. Department of Land Economy, University of Cambridge.

Villegas, C. y Chávez, C. (2004). "Costos de cumplimiento y poder de mercado: Aplicación al programa de compensación de emisiones". Cuadernos de Economía, Vol. 41, № 122: 91-123. 


\section{ANEXO 1}

Comparativo fuentes PARTiCiPANdo En EL PCE: 1999 Y 2006

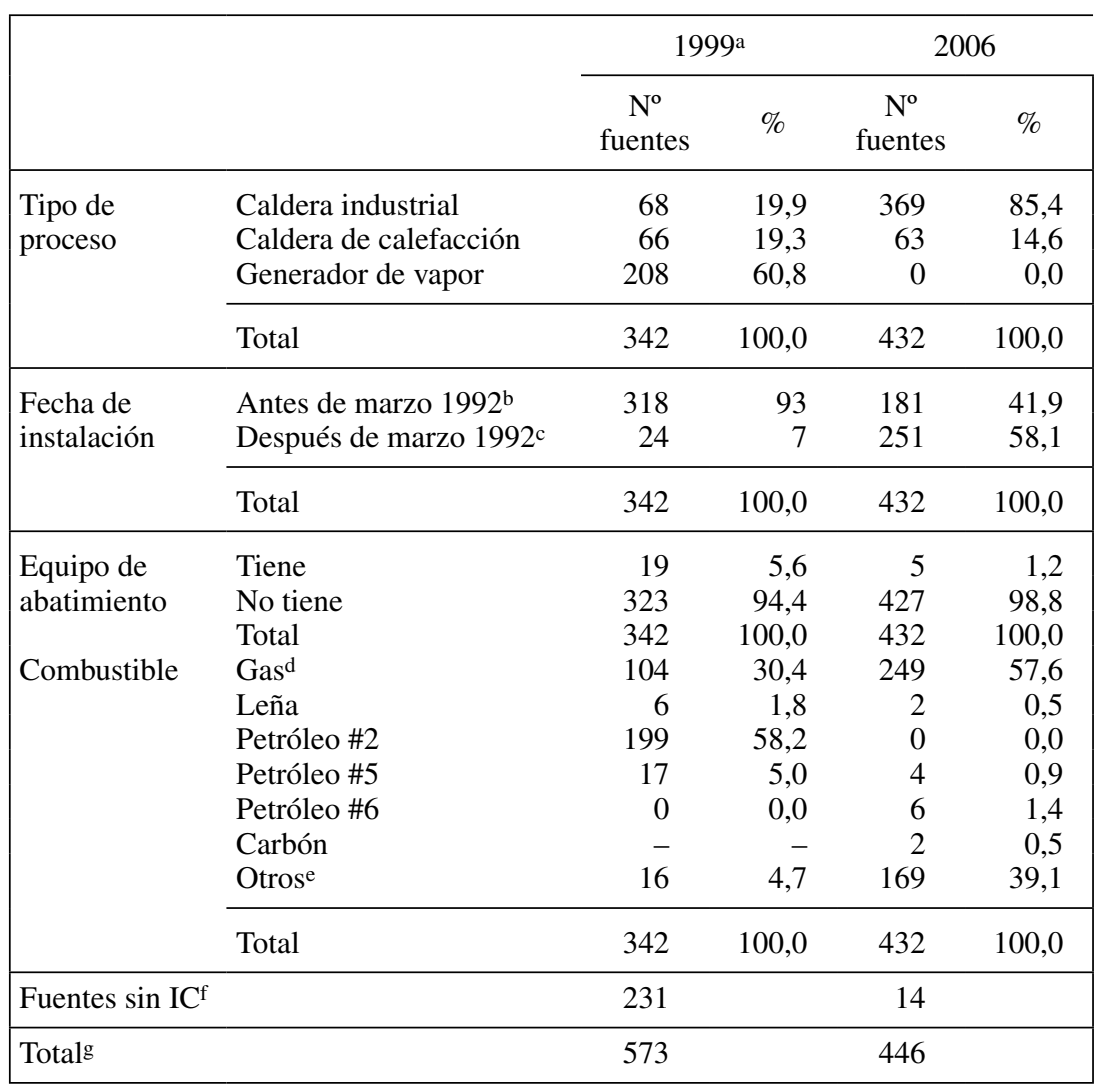

Fuente: Elaboración propia a partir SESMA (2007).

a Ver Palacios y Chávez (2002).

b Las fuentes instaladas antes de marzo 1992 son consideradas como las fuentes existentes, todas han recibido un nivel de EDI (Emisión Diaria Inicial) al año 1992 debido a la implementación del Programa de Compensación de Emisiones (PCE), establecido por el Decreto Supremo $\mathrm{N}^{\circ} 4$ (DS 4).

c Las fuentes instaladas después de Marzo 1992 son dichas nuevas, no tienen EDI sino un nivel de EDP (Emisión Diaria Permitida).

d Gas natural, gas de cañería y/o gas licuado.

e Aserrín, kerosene, lef, superlef, diesel, LMFO.

f Total de fuentes sin información completa.

g Total de fuentes participando en el PCE, considerando a todas las fuentes (con o sin información completa). 


\section{ANEXO 2}

\section{DETERMINACIÓN PROBABILIDAD DE MONITOREO Y COSTO DE FISCALIZACIÓN EN UN SISTEMA DE ESTÁNDARES DE EMISIÓN}

Aquí se detalla la metodología de determinación de la probabilidad de monitoreo y los costos de fiscalización de una política de comando y control bajo tanto bajo supuesto de información completa como incompleta.

La probabilidad de monitoreo de cada fuente es calculada como se describe a continuación:

$$
\begin{gathered}
\pi_{i}=\operatorname{Max}\left(\pi_{i, j}\right) \\
\pi_{i, j}=\frac{C\left(e_{i, j}^{*}\right)-C\left(e_{i, j}\right)}{f^{*}\left(e_{i, j}-s_{i}\right)}
\end{gathered}
$$

(2.1-a) Con

Para $\forall i, j$ tal que:

$$
e_{i, j}>s_{i} \geq e_{i, j}^{*}
$$

Donde:

$i=$ Índice de fuentes reguladas $(i=1, \ldots \ldots, N)$.

$j=$ Índice de opciones de control $(j=1, \ldots, M)$.

$C\left(e_{i, j}^{*}\right)$ : Costo de abatimiento asociado al nivel de emisiones optimal de la fuente $i$ en M\$/año.

$C\left(e_{i, j}\right)$ : Costo de abatimiento de la fuente $i$ utilizando la opción $j$ en M\$/año.

$e_{i, j}^{*} \quad:$ Nivel de emisiones optimal de la fuente $i$ utilizando la opción $j$ en kg/año.

$e_{i, j} \quad:$ Nivel de emisiones de la fuente $i$ utilizando la opción $j$ en $\mathrm{kg} / \mathrm{año}$.

$s_{i} \quad:$ Meta individual de la fuente $i$ en $\mathrm{kg} / \mathrm{año}$.

$f \quad$ : Multa impuesta por el regulador en $\mathrm{M} \$$.

Por lo tanto, el costo agregado de fiscalización del sistema de estándares de emisión bajo el supuesto de información completa está dado por: 
$(2.2-a)$

$$
C F_{1}^{E E}=w *\left[\pi_{1}+\pi_{2}+, \ldots \ldots \ldots \ldots \ldots, \pi_{N}\right]
$$

Con información incompleta, el costo agregado de fiscalización está expresado como sigue:

$$
C F_{2}^{E E}=w^{*} \operatorname{Max}\left(\pi_{i}\right) * N, \quad i=1, \ldots, N
$$

